\title{
Pratiques
}

Linguistique, littérature, didactique

$159-160 \mid 2013$

Le figement en débat

\section{Étude de cas : soi-disant}

\section{Corinne Féron et Danielle Coltier}

\section{(2) OpenEdition \\ Journals}

Édition électronique

URL : http://journals.openedition.org/pratiques/2843

DOI : $10.4000 /$ pratiques. 2843

ISSN : 2425-2042

\section{Éditeur}

Centre de recherche sur les médiations (CREM)

\section{Édition imprimée}

Date de publication : 15 décembre 2013

\section{Référence électronique}

Corinne Féron et Danielle Coltier, «Étude de cas : soi-disant », Pratiques [En ligne], 159-160 | 2013, mis en ligne le 30 juin 2016, consulté le 03 mai 2019. URL : http://journals.openedition.org/pratiques/2843 ; DOI : 10.4000/pratiques.2843 


\title{
Étude de cas : soi-disant
}

\author{
Corinne Féron
}

Université du Mans et 3L.AM

Danielle Coltier

Université du Mans et CREM

Nous envisagerons la notion de figement à partir de l'exemple de soi-disant, sous deux angles : d'une part, le figement comme propriété d'une séquence de mots dont chacun a par ailleurs un fonctionnement autonome, ce qui amènera à examiner les caractéristiques de la séquence en question par rapport aux séquences libres ; d'autre part, le figement comme processus intervenant dans le changement linguistique :

- dans les études diachroniques sur la grammaticalisation, le figement est généralement défini comme une coalescence, une soudure d'éléments d'abord indépendants (ex. beau coup > beaucoup ; $f$. par exemple Marchello-Nizia $2006: 41$ ); il n'est donc que l'un des (nombreux) processus à l'œuvre dans le changement, et il concerne exclusivement le plan de la syntaxe ;

- cette notion reçoit dans d'autres études une définition plus large : ainsi c'est, selon Lecolle (2006:23), une «"fixation" de la forme et/ou du sens de séquences discursives dans la compétence discursive collective, voire en langue $\gg{ }^{(1)}$.

Cependant, même si l'on adopte la définition « large », tous les changements qui interviennent dans la formation de l'unité soi-disant ne peuvent être ramenés au figement; il s'agira donc d'examiner les relations que le figement entretient avec les autres processus, et de tenter d'établir une «chronologie logique » de ces divers changements.

Avant d'aborder la question du figement, nous proposerons une description

(1) De même, Perrin (2011:82) définit le figement comme un processus « portant indifféremment sur l'application de n'importe quel ensemble de règles syntaxiques, morphologiques ou sémantiques initialement associées à une expression. » 
des emplois de soi-disant (désormais SD) en français contemporain, dans le cadre théorique des univers de croyance de R. Martin.

Le corpus est constitué à partir de deux bases textuelles, 7FMR et Frantext. Nous avons par ailleurs procédé à des requêtes sur Internet.

\section{Description des emplois de soi-disant en français contemporain}

Dans tous les cas, SD signale qu'il y a emprunt à un autre discours — SD introduit donc l'image d'univers d'un énonciateur ${ }^{(2)}$ — et signifie une réserve du locuteur vis-à-vis de cet autre discours ; ainsi, les énoncés suivants paraissent difficilement acceptables :

(1) ' Luc, soi-disant malade, est très mal en point (SD signale que l'assertion « Luc est malade » est au moins douteuse pour le locuteur, alors que « Luc est très mal en point », argumentativement orienté vers la conclusion « Luc est malade », est pris en charge — au sens de Martin $1987^{(3)}$ — comme vrai par le locuteur).

(2) 'Son soi-disant sauveur lui a évité la mort.

(3) 'Il a soi-disant passé ses vacances en Grèce ; les photos qu'il y a faites sont superbes.

SD est donc un modalisateur : il dit quelque chose de la prise en charge de cet autre discours par le locuteur. A savoir : le locuteur juge que la prise en charge comme vrai du contenu propositionnel par l'énonciateur rapporté est au moins douteuse/discutable. Autrement dit, SD indique que dans l'univers de croyance du locuteur, asserter « Luc est malade », pour (1), « un tel est son sauveur » pour (2) et «il a passé ses vacances en Grèce » pour (3) est douteux, et que le contenu propositionnel doit être affecté d'une valeur autre que «vrai». En bref, SD signifie que le locuteur discute l'affectation de la valeur « vrai » au contenu propositionnel que supposent ces assertions.

Selon son insertion dans la phrase, SD est classé soit comme adverbe, soit comme adjectif.

\subsection{SD adverbe}

Du point de vue sémantique, SD a toujours un fonctionnement exophrastique : les exophrastiques ont pour caractéristique d'être « des constituants externes à la phrase, en ce sens qu'ils ne participent pas à la construction de son sens référentiel, mais représentent des traces de l'intervention du locuteur, qui commente tout ou partie de son énoncé ou de l'acte qui le produit » (Guimier 1996 : 6, gras

(2) «Il y a image d'univers dès lors que, épistémiquement, le locuteur renvoie, dans son discours, à un univers de croyance. Il en est ainsi quand le locuteur évoque un hétéro-univers », par exemple «l'univers d'un énonciateur dont est rapporté le dire (Il affirme, il pense, il s'imagine... que p) [...]» (Martin 1992:47).

(3) Pour qui prendre en charge équivaut à attribuer une valeur de vérité ( $c f$. Martin 1987: 11 et $1992: 43)$. 
de l'auteur). SD fait partie des assertifs, qui « discutent de la valeur de vérité de l'énoncé » (Guimier 1996 : 112), et plus précisément, selon Nøjgaard (1995: 236), de la sous-classe des adverbes polyphoniques (de même que censément, prétendument, dit-on, de notoriété publique) : SD «manifest[e] l'intervention de la tierce personne : la voix qui parle à travers le participe disant n'est pas identique à celle du locuteur ».

Du point de vue syntaxique, SD est incident à une phrase :

(4) Nous sommes tous là à nous mystifier les uns les autres, et personne ne sait pourquoi. Soi-disant on sacrifie à la pureté : mais où est-elle, la pureté ? Qu'on me la montre! (Beauvoir, Les Mandarins, 1954)

ou incident à un syntagme (éventuellement réduit à un mot), que l'on considérera comme une phrase tronquée ${ }^{(4)}$, par exemple dans :

(5) [...] les moines mendiants, soi-disant nomades mais réglés comme des bureaucrates du fisc. (Ollivier, L'Orphelin de mer, 1982)

SD est incident à la phrase tronquée « les moines sont nomades ». On représentera ce type d'emploi par $N / G N S D Y$, N représentant un nom éventuellement expansé. $N S D Y$ correspond au cas où $S D Y$ est déterminatif par exemple dans " c'est un comble pour une mairie soi-disant de gauche », GN SD Y au cas où $S D$ $Y$ est en apposition, par exemple dans (5). Quant à Y, il s'agit d'un groupe nominal (« Le premier, à Montreuil, est un L.E.P., soi-disant un lycée d'enseignement pratique ; en fait, c'est une école professionnelle », Dolto, La Cause des enfants, 1985), d'un groupe adjectival (en 5 par exemple) ou d'un groupe prépositionnel («Un jour, mon frère voulut faire un gâteau soi-disant de son invention », Mréjen, Mon Grand-Père, 1999).

\subsection{SD adjectif}

Placé entre un déterminant et un nom ${ }^{(5)}$, (Dét. $S D N$, où $\mathrm{N}$ représente un nom, comme en 6 , éventuellement expansé, comme en 7) :

(6) Deux défilés différents vont se dérouler demain $1^{\text {er }}$ Mai - un organisé par les soidisant communistes, l'autre par la C.F.D.T., le P.S.U. et diverses organisations gauchistes. (Manchette, Journal 1966-1974, 2008)

(7) Comme ces phrases en disent long sur les soi-disant élites du temps [...]! (Sollers, Le Secret, 1993)

(4) Cf. Guimier (1996: 104, note 1). Guimier (1988: 273 et 276) donne une analyse plus conforme à la théorie guillaumienne de l'incidence : l'adverbe étant caractérisé par son incidence externe du second degré, soit son incidence « affecte celle-ci [la phrase] de l'extérieur, se donnant pour support, globalement et indistinctement, les différentes incidences qui structurent l'énoncé phrastique ", soit elle «échoit, de l'extérieur, à l'ensemble des incidences immanentes à un syntagme déjà réalisé ».

(5) SD peut aussi figurer — du moins, si l'on suit Riegel e.a. (2009:313 et 661) — devant un GN sans déterminant, après la préposition en : «les sociétés industrielles [...] éprouvent toujours le besoin d'agir en soi-disant père vis-à-vis de ces populations du Tiers-Monde [...] » (Dolto, La Cause des enfants, 1985). 
SD fonctionne comme un " adjectif inclassable » ou « du troisième type » (Schnedecker 2002 ; Riegel e.a. 2009 : 634-638), dénominations qui étiquettent un mode de fonctionnement de la classe des adjectifs.

a) Ces adjectifs sont caractérisés par des restrictions formelles :

- Ils sont non gradables (comme les relationnels).

- Ils ne s'emploient qu'en fonction d'épithète, sauf exception.

— Ils sont «généralement antéposés au nom » (Riegel e.a. 2009 : 599), ou, s'ils peuvent être postposés, ils sont alors en emploi qualificatif (Riegel e.a. 2009 : 636).

- Ils ne peuvent pas être coordonnés à un adjectif qualificatif.

— «Ils ne se doublent pas d'un nom de propriété » (Riegel e.a. 2009 : 635) ;

Schnedecker (2002: 10) signale que font exception les adjectifs de comparaison (analogue, égal...) et que si d'autres adjectifs « du troisième type » ont des dérivés nominaux, ceux-ci « ne s'interprètent pas comme noms de qualité, au même titre que blondeur ou gentillesse ».

b) Du point de vue sémantique, «ces adjectifs ont en commun de ne pas modifier le nom dont ils sont épithètes par spécification ou sous-catégorisation, comme le font les deux autres catégories d'adjectifs [qualificatifs et relationnels]. Ils fonctionnent plutôt comme des modalisateurs de la valeur référentielle du nom dont ils sont épithètes " (Riegel e.a. 2009 : 635); ainsi, dans la suite Dét. $S D N$, SD signale que $\mathrm{N}$ est une dénomination empruntée par le locuteur et que :

- soit cette dénomination est, aux yeux du locuteur, non pertinente, " inadéquate au réel » (Authier-Revuz 1995 : 361) ; autrement dit, dans par exemple « le soi disant artiste », l'assertion sous-jacente "celui-ci, qui se dit/est dit artiste, est un artiste » est au moins douteuse aux yeux du locuteur (n'est pas prise en charge comme vraie par le locuteur);

- soit le référent du GN (dét $S D N$ ) n'a pas les qualités attendues du stéréotype de $\mathrm{N}$; par exemple dans : « Je suis allé chez le docteur X. Ce soi-disant médecin ne m'a pas même ausculté », le titre/la profession médecin est bien adéquat(e) au réel ; ce qui est en cause, c'est le stéréotype associé au nom médecin, ici, un procès (« un médecin ausculte son patient »).

Cependant, dans quelques rares occurrences, SD s'éloigne de l'emploi d'adjectif du troisième type et se rapproche de l'emploi qualificatif :

— SD postposé semble dénoter une propriété du référent (au même titre que feint, apparent, supposé, illusoire...) :

(8) ce que je veux dire et tu ne pourrais le nier si tu voulais te souvenir avec moi, / ce que je veux te dire, / tu ne manquais de rien et tu ne subissais rien de ce qu'on appelle le malheur. [...] / Nous nous surveillions, / on se surveillait, nous nous rendions responsables de ce malheur soi-disant. / Parce que tout ton malheur ne fut jamais qu'un malheur soi-disant, / tu le sais comme moi je le sais [...] (Lagarce, Juste la fin du monde, 2000) ${ }^{(6)}$

(6) Une interprétation de SD comme adverbe modifierait considérablement l'interprétation : soi-disant serait incident, dans la première occurrence, à l'ensemble « nous nous rendions responsable de ce malheur », et dans la seconde, à «parce que tout ton malheur ne fut jamais 
- SD fonctionnant comme adjectif du troisième type peut être coordonné à un adjectif du même type (exemples relevés sur Internet: le dit (et soi-disant) $N$, le soi-disant ou vrai $N$, le soi-disant ou supposé $N . .$.$) mais aussi à un adjectif quali-$ ficatif ; dans (9), il s'agit d'un adjectif subjectif (affectif) :

(9) Cette réflexion, inépuisable bien sûr mais dont on ne peut dire qu'elle ne soit pas d'actualité en face d'une soi-disant mais bien inquiétante "post-modernité » (http://www.ey.asso.fr/ edito_8.htm)

- On trouve (sur Internet uniquement) des occurrences d'un dérivé nominal, soi-disance (interprétable, peut-être, comme « fait de se dire sans raison/à tort pourvu de telle qualité ») :

(10) ben alors le ministre fais passer la fnaim et les notaires il a bien compris, comme tout ceux qui n'ont de socialiste que la soi-disance, qu'il vaut mieux conforter [...] (www.zanzimmo.com/immobilier/les-notaires_p5.html)

\subsection{SD nom}

SD est exceptionnellement attesté comme nom, sans que l'on puisse, au vu des quelques occurrences relevées, lui attribuer un sens stable; dans (11), il s'agirait d'un faux-semblant, d'une apparence trompeuse ou d'un on-dit :

(11) Toulon est une ville qui est riche de jolis endroits accaparant l'intérêt des étrangers, des touristes locaux et autres. Vu l'intensité de mouvement de déplacements, surtout grâce à son emplacement et à tout ce qu'il est en mesure d'offrir à ses visiteurs, faire des rencontres à Toulon n'est pas un soi-disant mais une réalité. (http://www.netnoo.com/guide/ rencontre-toulon)

\section{Soi-disant : une séquence figée en français contemporain ?}

2.1. Du point de vue morphosyntaxique, le figement de SD est repérable d'emblée.

- D'une part, du fait de sa construction, qui relève d'une syntaxe ancienne : soi devant une forme en -ant, qui a été remplacé par se en français préclassique ${ }^{(7)}$, s'est maintenu ici; SD fait partie des «blocs erratiques », «éléments ou constructions qui remontent à un état de langue antérieur. Ces éléments ont gardé leur syntaxe d'origine et apparaissent de ce fait comme extérieurs au système actuel »(Gross 1996:22).

— D'autre part, par la présence du trait d'union, indiquant qu'on a affaire à une unité linguistique, ce qui exclut toute manipulation, à savoir :

a) l'insertion ;

b) la coordination d'un des éléments à un autre mot : la coordination de $s o i$

qu'un malheur », que le locuteur ne prendrait donc pas en charge comme vrais ; or, le cotexte laisse entendre le contraire.

(7) Cf. Combettes \& Marchello-Nizia (2010:136). Si la forme forte est encore attestée au XVII ${ }^{\mathrm{e}}$ siècle, cet emploi est rare et fait figure, selon Fournier (1998: 79), "d'archaïsme ». 
semble exclue $(*$ « les autres et soi-disant»; « soi et les autres disant » est possible, mais on aurait alors la forme en -ant de dire) ; " Luc, soi-disant et croyant artiste» (où soi serait en facteur commun) parait étrange, alors que disant peut être coordonné à une autre forme en - ant, avec se en facteur commun : "Luc se disant et croyant artiste », et que l'ensemble soi-disant peut être coordonné à se + forme en -ant (avec « cultivées» en facteur commun dans 12) :

(12) Beaucoup de gens soi-disant et se disant cultivées sont en train de prendre activement part [...] à ce génocide [...] (http://fr.scribd.com/doc/ 99039538/DLEP-LETTRE-31-10-2010)

où l'on pourrait interpréter soi-disant comme «d'après ce qu'on dit » vs se disant (eux mêmes).

c) la substitution d'un synonyme de dire (* « le soi-nommant médecin »; * « Luc soi-nommant/appelant médecin ») ; on relève certes soi-proclamant:

(13) [...] dans une nouvelle qui porte pour titre l'hégélien, raconte l'aventure d'un soidisant, soi-proclamant disciple de Hegel qui, démocrate révolutionnaire, rentre en Allemagne pour s'y battre contre la réaction, en 1849. (D'Hondt, La réception profane de Hegel en France, Google books)

mais il nous semble qu'il s'agit moins d'une substitution de synonymes que d'une forme analysable comme relevant du défigement, en ce sens que soi + forme en -ant ne relève pas de la syntaxe du français actuel ;

d) la substitution à soid'un autre pronom. Il y a peu de cotextes où soi peut être remplacé par un autre pronom dans l'usage courant en français contemporain; on citera tout de même : il « s'expliquait trop bien [...] que le comte fût à peine mâ̂tre de soi » (Bourget, dans NPR), où lui serait possible, mais créerait une ambiguïté. On signalera cependant que l'on aurait « Toi, te disant/ sachant/ croyant/ nommant.. » mais « Toi, soi-disant» :

(14) toi, soi-disant « citoyen », soi-disant « engagé », donne allègrement dans le négationnisme ????? [réponse au message d'un internaute qui apparaît sous le nom de « le citoyen engagé »] (http://lecitoyenengage.fr/ 2012-sera-t-elle-lannee-de-la fin-du-mensonge-juif-1144)

autrement dit, on a la forme de troisième personne, soi, même si SD peut se gloser (ici, à partir du contexte) par : « qui te dis (à tort/sans raison)».

2.2. Du point de vue du sens, on l'a signalé, SD signifie que le locuteur introduit le discours d'un énonciateur, discours vis-à-vis duquel il émet une réserve, alors que par exemple «Luc, se disant (soi-même) malade/disant de lui qu'il est malade » (vs « Luc, soi-disant malade ») ou « un tel se disant son sauveur» (vs « son soi-disant sauveur ») ne dit rien de l'attitude du locuteur vis-à-vis de « Luc est malade » ou de « un tel est son sauveur »; en ce sens, soi-disant est une séquence opaque.

Mais, si SD renvoie toujours à un autre discours auquel le locuteur n'adhère pas, il a deux usages : un usage qui privilégie une interprétation réflexive (Rosier 1999 : 198 parle d'« usage réflexif»), quand le $\mathrm{N}$ désigne un humain ou peut y ré- 
férer indirectement/métonymiquement ; un usage clairement non réflexif : le $\mathrm{N}$ ne désigne pas un humain et une référence indirecte à un humain n'est pas plausible.

a) Usage privilégiant une interprétation réflexive : le discours rapporté est le discours d'un énonciateur sur soi-même; dans cet usage, seul accepté par la norme, SD peut être glosé par « qui se dit lui-même (à tort/sans raison aux yeux du locuteur) »; il est exemplifié par l'emploi adjectival dans la suite Dét $S D N$ («Le soi-disant plombier était un cambrioleur», NPR) et par l'emploi adverbial dans la suite $N / G N S D Y$, où l'adverbe SD est incident à une phrase tronquée (voir supra) ; par exemple dans «L'enfant, soi-disant malade » ${ }^{(8)}$, l'énonciateur est le référent du GN, «l'enfant», et ce référent est nécessairement un être doué de parole (être de parole auquel il peut être fait référence métonymiquement comme dans : « un comble pour une mairie soi-disant socialiste !»)

b) Usage non réflexif : l'autre discours n'est pas le discours d'un énonciateur sur soi-même. Cet usage est exemplifié par « un soi-disant palace », « un hôtel soi-disant luxueux » (« un établissement qui est dit/appelé (à tort) palace », « un hôtel qui est dit (à tort) luxueux ») ; l'énonciateur ne saurait être le référent du GN. Favorisé par la présence d'un nom qui ne dénote pas un être de parole, cette interprétation connaît d'autres cas de figure ; en effet, en présence d'un $\mathrm{N}$ /animé humain/, l'interprétation selon laquelle le dire serait celui du référent du GN n'est pas systématique ; dans "Cet homme, soi-disant attardé », on attribuera plus difficilement — pour des raisons pragmatiques — «attardé» au discours du référent de « cet homme» ( $c f$. Goosse 2008: 858-859: « il est un peu trop simple d'opposer les choses aux personnes. Même pour des personnes, soi-disant peut heurter la logique sourcilleuse », par exemple dans « des autres soi-disant incendiaires du Reichstag », Malraux, Antimémoires). Dans cet usage non réflexif, soit le cotexte permet d'attribuer une source au dire — dans l'exemple (15), c'est le référent de ce pourri:

(15) Et pourquoi qu'il avait un flingue, ce pourri? Pour défendre quoi ? sa bagnole? sa D.S. soi-disant fiable? (Blier, Les Valseuses, 1972)

soit il ne le permet pas ; dans ce cas, SD introduit ce que Rosier (1999: 181) appelle un « on dit », et Noailly (1999: 62), pour le cas où, selon le présent article, SD est adverbe dans la suite $N / G N S D Y$, la voix « de la doxa, responsable du choix d'un adjectif stéréotypé ».

Dans les autres types d'emplois à priori rejetés par la norme, on peut considérer que SD est en usage réflexif dès lors que SD introduit le discours d'un énonciateur sur lui-même, concernant par exemple ses propres intentions; on a un discours d'un énonciateur sur soi :

(16) Il a travaillé tout l'été, soi-disant pour s'acheter une nouvelle batterie. (Manoeuvre, L'Enfant du rock, 1985) [où l'assertion peut être interprétée comme un dire du référent de «il»].

(8) Ici, une interprétation non réflexive (« dont on dit qu'il est malade ») est également possible. 
de même pour $S D$ que ${ }^{(9)}$ dans :

(17) Voilà le barjot qui décanille ! Soi-disant qu'il est allé chercher quelque chose que Jeanne aurait laissé chez lui. (Dorin, Les Vendanges tardives, 1997) [« le barjot dit de lui-même que...»]

Mais le dire peut ne pas concerner l'énonciateur auquel est attribuable ce dire ; il s'agit alors du discours d'un énonciateur sur autre que soi :

(18) Nous avons regagné l'aérodrome dont le bar servait de rendez-vous à l'élite européenne parce qu'on dominait la ville de quelques mètres et que, soi-disant, la température était moins torride : elle me parut aussi accablante qu'en bas (Beauvoir, La force des choses, 1963) [l'énonciateur pourrait être assimilé à «l'élite européenne»]

(19) Mais vous-même, parlez devant lui de choses qui vous intéressent, au lieu d'essayer de le faire parler de ce qui, soi-disant, devrait l'intéresser. (Dolto, La Cause des enfants, 1985) [que l'on pourrait interpréter par exemple comme « des choses dont vous dites/certains disent qu'elles devraient l'intéresser »]

Au regard du sens, SD présente donc les propriétés d'une séquence figée, mais inégalement selon les usages : dans l'usage prescrit par la norme — l'usage réflexif — SD reste partiellement compositionnel, puisqu'il s'agit d'un dire sur soimême ; autrement dit, cet usage maintient le «sens ascendant» (le « sens construit originel») de la séquence (Perrin 2011 : 83). Il est à noter que les formes issues d'un défigement relevées sur Internet sont construites sur cette interprétation; on a cité ci-dessus en (13) soi-proclamant (《se proclamant soi-même »), juxtaposé à soi-disant; on peut ajouter :

(20) «J'admirerai ton conte si tu prises mon tableau», on ne sort pas de ces marchés sordides, qui font pulluler les soi-croyant artistes, et sapent les jugements de valeur [...] (http://diarrhy.centerblog.net/ 9-une-ecoute-projective-et-narcissique)

(21) Pire, nos sociétés « postindustrielles », soi-disant et soi-pensant « civilisées », fonctionnent sur le modèle de la spécialisation sociale [...] (INFORMETHIC_ARCHIVES_2009-2012_FINAL.pdf)

De même, autant que l'on puisse lui attribuer un sens, le dérivé soi-disance ( $c f$. supra en 10) renvoie à un dire sur soi.

(9) Soi-disant que est ordinairement considéré comme une locution conjonctive, «populaire » ou «familière »; $c f$. par exemple Rosier (1999: 179), le TLF (sv. soi-disant), le NPR et le Lexis. Goosse (2008: 1445) considère que «par rapport à la phrase ou à la sous-phrase précédente, soi-disant que peut indiquer une cause prétendue $»$. 


\section{Le figement en diachronie}

\subsection{Description de la forme de départ}

Parler de figement, c'est supposer comme point de départ une séquence qui relève de la syntaxe libre ( "générée par les règles combinatoires mettant en jeu à la fois des propriétés syntaxiques et sémantiques », Gross 1996 : 6).

Nous donnons ici une description des occurrences de moyen français ${ }^{(10)}$ (nous n'avons pas trouvé d'occurrence en ancien français) ; elles figurent d'abord et majoritairement dans des textes juridiques (des registres d'audience), quelquesunes dans des textes à caractère historique ou politique, d'autres enfin, peu nombreuses, dans des textes didactiques et littéraires.

\subsubsection{Plan morphologique}

La forme forte soi alterne avec d'autres formes fortes (toujours de personne 3 ou 6 dans le corpus) :

- exceptionnellement, avec lui après un GN singulier :

(22) LEQUEL GUILLAUME, disant lui estre remenbrant des extorcions, mauvaistiez et larrecins par lui commises [...] (Registre criminel du Châtelet t.1,1389-1392)

— fréquemment, avec eux après un GN pluriel :

(23) $[\ldots]$ et donnez par les dessus nommez conseilliers, eulx disans commissaires de la Court en ceste partie [...] (Fauquembergue, Journal t.2, 1421-1430)

ou après une énumération valant idée de pluriel :

(24) Jaquet Alargent, Charlot du Moustier, Herbert Boursaut, Morinot Fale, Jaquet Hainselin, Pierre Le Menestrat, eulz disans varlés de chambre, Jehan Fourqueval, Blancardin Thierry, Jaquet Marcade, eulz disans sommeliers, Pierre Godet, sommelier du linge [...] (Fauquembergue, Journal t.2, 1421-1430)

Ces alternances soi/lui/eux sont observées d'une façon générale dans l'emploi du pronom avec une forme en -ant ou un infinitif ${ }^{(11)}$.

\subsubsection{Plan syntaxique}

Le pronom, le plus souvent antéposé à disant, peut cependant lui être postposé (cf. supra 22 : «disant lui estre remembrant»); il peut en être séparé, notamment par un adverbe :

(25) Charles, soy nagaires disant dauphin (Fauquembergue, Journal t.2, 1421-1430)

(10) Nous adoptons la périodisation suivante : moyen français, 1330-1550 ; français préclassique, 1551-1660; français classique, 1661-1800.

(11) «[...] le réfléchi est de très loin le plus fréquent au singulier; au pluriel se marque une hésitation, mais le non-réfléchi semble dominer » (Marchello-Nizia 1979 : 198). 
Quant à disant, il peut être coordonné à une autre forme en -ant :

(26) $[. .$.$] le commandement de la voix puerile soy disant et renommant du lignage des$ nobles seigneurs barons et chevaliers de vostre maison de Monfalcon et de Flascieu [...] (Antitus, Poésies, c.1500)

\subsubsection{Plan de l'interprétation}

Le $D M F$ mentionne $\mathrm{SD}^{(12)}$ parmi les emplois pronominaux de dire suivi d'un attribut, et lui consacre par ailleurs un article ; selon cet article, SD introduit dans tous les cas le dire d'une personne sur soi, mais deux définitions sont données : l'une correspond au cas où le locuteur doute de ce dire (« qui prétend être tel (qu'on ne reconnaît pas pour tel) »), l'autre, au cas où il ne lui accorde pas de valeur de vérité («qui dit être tel (qu'on n'a pas vérifié être tel)»). Il nous semble que la première définition ne correspond qu'à une interprétation, construite à partir du cotexte ou de connaissances extralinguistiques.

En effet, rien ne permet, au vu des occurrences de moyen français, d'interpréter SD autrement que comme une marque de discours rapporté — donc, en l'occurrence, autrement que comme « qui se dit» :

- SD introduit une image d'univers, celle d'un énonciateur assimilable au référent du contrôleur de disant; dans cette image d'univers, l'assertion rapportée, par exemple pour (23) ci-dessus : « les dessus nommez conseilliers sont commissaires de la Court en ceste partie », est présentée comme vraie ; - cette assertion rapportée n'est en revanche pas prise en charge par le locuteur : celui-ci ne lui attribue pas de valeur de vérité.

Comme pour tout discours rapporté avec le verbe dire, le cotexte ou des connaissances extralinguistiques peuvent permettre d'interpréter l'attitude du locuteur vis-à-vis de l'assertion rapportée :

- cette assertion est douteuse pour le locuteur :

(27) $[\ldots]$ celui ne doit point « estre » reputé noble se avecques la noblesse de la chair il n'a noble pensee; et tout noble abhorre chose deshonneste et pechié. Maiz aucuns, eulx disans nobles, ont tellement accoustumé de faire le contraire de noblesse, que, par leurs manieres de faire, il semble que ung homme n'est point noble, se ilz n'est abhominable pecheur, et s'i ne maulgree et regnie et jure Dieu, et face toutes choses detestables a reciter (Juvénal des Ursins, Tres reverends et reverends peres en Dieu, 1433)

Le cotexte indique clairement que les référents de aucuns n'ont pas les propriétés impliquées - selon le locuteur, qui s'appuie sur une autorité qu'il cite auparavant - par l'adjectif noble, quand bien même ils sont ainsi qualifiés et qualifiables du fait de leur naissance ; cette qualification qu'ils se donnent — de façon «juste» au regard de leur naissance — est discutable au regard de ce que doit être la noblesse selon le locuteur.

(12) Par commodité, nous continuons d'utiliser pour le moyen français l'abréviation SD, alors que l'on peut avoir un pronom de forme forte autre que soi, et que, selon nous, on n'a pas affaire, dans cet état de langue, à une unité. 
Dans :

(28) Comment Guillaume roy d'Engleterre et soy disant duc de Normendie filz de Guillaume le Bastart dit nothus eult de fait la duchié de Normendie et l'osta a Robert son frere, et depuis par orgueil voult avoir la duchié contre le roy et qui pis le royaulme de France, par quoy il perdit le droit de Normendie, s'aulcun en y avoit (Juvénal des Ursins, Tres Crestien..., c.1446)

soy disant parait glosable par « se déclarant/proclamant duc de Normendie ", donc comme une sorte de performatif qui serait rapporté par le locuteur, la légitimité du titre « duc de Normandie » étant mise en cause par le cotexte droit, qui évoque une usurpation.

On notera que même en l'absence de cotexte permettant de construire une interprétation, la présence de SD devant un nom désignant un titre important et supposant une reconnaissance générale, indique qu'il n'y a pas unanimité sur ce titre :

(29) Hier, furent leues certeinnes lettres que monseigneur le duc d'Orleans avoit ordonné à envoier à Henry de Lanclaste, soy disant roy d'Angleterre [...] (Baye ; Journal, t.1, 1400-1410, 75)

On pourrait comprendre, comme dans (27), que c'est le comportement du référent du GN qui est en cause - il ne serait pas celui qu'on attend d'un roi d'Angleterre ; cependant, des connaissances historiques nous conduisent à interpréter le recours au discours rapporté comme une remise en question de la légitimité de l'attribution du titre de roi d'Angleterre à Henri de Lancastre.

- Dans de nombreux cas cependant, et notamment dans les registres tenus par des greffiers, rien ne permet d'interpréter SD comme une marque de réserve ; il arrive même que le cotexte indique que le locuteur prend en charge comme vraie l'assertion rapportée ; par exemple, dans :

(30) Neantmoins Thierry L'Enfant, Henry et Guillaume dits les Flamen, Jehan de Lourme, Guillaume Fortin, eulx disans sergens et forestiers des bois du dit chevalier, et Jehan de Riville, bastart, escuier, naguaires vindrent à la dite maison du dit Galopin [...], lesquieulx sergens, forestiers et bastart crierent et demanderent que l'on leur ouvrist l'uis de la dite maison [...] (Le Canarien, Pièces justificatives, $1327-1470$ [1395])

la reprise de sergens et forestiers dans un groupe nominal comprenant un déterminant anaphorique (lesquieulx), au même titre que bastard - qui est, dans sa première occurrence, en apposition au nom propre Jehan de Riville - laisse entendre que l'assertion « Jehan de Lourme, Guillaume Fortin sont sergens et forestiers » est vraie pour le locuteur.

\subsubsection{Bilan sur SD en moyen français}

En moyen français, dans la suite $N / G N S D Y$ (où Y peut être un $\mathrm{N}$ ou un GN, un groupe adjectival, par exemple en 27, un groupe prépositionnel en à ou de, comme en 26 et infra, en 31, un groupe infinitif enfin, comme en 22), on a la forme en -ant de dire - d'où les variations possibles dans la forme du pronom - 
qui réfère à un procès, éventuellement situé dans le temps (cf. supra 25 : « soy nagaires disant dauphin »). Cette forme se fléchit régulièrement si le GN qui précède est au pluriel ( $c f$. supra 23 et 27) ou s'il y a une énumération de GN comme c'est le cas en (24) et (30) (nous n'avons pas d'exemple avec un $\mathrm{N}$ féminin).

\section{a) Construction}

On peut considérer que l'on a dans $S D Y$ une des constructions du verbe dire, à savoir une «proposition infinitive de type savant ${ }^{(13)}$, le plus souvent elliptique de estre, comme elle peut l'être avec d'autres formes de dire ${ }^{(14)}$, le «sujet » de la proposition infinitive (le pronom de forme forte) étant en relation de coréférence avec le contrôleur de disant ${ }^{(15)}$.

b) Insertion du groupe $S D Y$ dans la phrase

Le plus souvent, $S D Y$ est un groupe (dont disant est le mot tête) modifieur du contrôleur de disant, c'est-à-dire d'un nom (si SD est épithète) comme, semble-til dans (31) :

(31) Jaques de Bailleul, serviteur soy disans du duc de Bourgoigne, est venu dire à la Court de par ledit duc que l'en ne procede point contre Olivier de Stannevelle ne ses compaignons, jusques à ce que l'en ait autre nouvelle. (Baye, Journal t.2, 1411-1417)

ou d'un GN (s'il est apposé, par exemple en 30) ${ }^{(16)}$.

Comme tout groupe dont la tête est une forme en -ant, SD Y peut aussi être placé non pas immédiatement après le $\mathrm{N}$ ou GN (contrôleur de disant), mais en fin de phrase, avec valeur circonstancielle (la préposition en n'étant pas obligatoire en moyen français) :

(32) ITEM, Thore, Dathan, et Abiron [...], orent envie sur leur prince Moyse et de ce que il avoit seigneurie sur le peuple, eulx disans plus sains et mieulx dignes de gouverner que lui. (Pizan, The "Livre de la Paix», 1412-1413)

Par ailleurs, en moyen français, le groupe comprenant une forme en -ant accompagnée de ses compléments peut être substantivé ( $c f$. par exemple : «comme il apparra plus plainement aux lisans et bien entendans ce present traittié », Bueil, Le Jouvencel t.1,1461 cité par Martin \& Wilmet $1980: 219)$ ). Ainsi, dans :

(33) $[\ldots]$ ung soy disant lieuxtenans des eaues et forestz [...] (Juvénal des Ursins $A, A$, $A$, nescio loqui, 1445)

(13) Exemples de "proposition infinitive savante » avec dire à un mode personnel : " et pour cela meismes il dit sapience estre chief de toutes les sciences » (Pizan, Le livre des fais et bonnes meurs du sage roy Charles V t.2, 1404). «Et pour ce que ledit homme se disoit estre au conte de Savoie » (Baye, Journal t.1, 1400-1410).

(14) «[...] un frere qui se dit cordelier [...]» (Registre criminel du Châtelet t.1, 1389-1392).

(15) Cette construction n'est pas propre à dire : " pour quoy je vous declaire que ledit Seigneur tres chrestien, mon souverain Seigneur invincible, qu'il quicte et renonce à toutes voz aliances, amitiez et confederations, soy declarant vostre ennemy mortel » (Marot, Le Voyage de Venise, 1526).

(16) Soit ce groupe suit immédiatement le $\mathrm{N}$ ou le GN, soit il en est séparé par un autre modifieur auquel il est coordonné ( $c f$. exemple 28) ou juxtaposé. 
ung peut aussi bien être déterminant (auquel cas le groupe « soy disant lieuxtenans des eaues et forestz » serait substantivé) que pronom (et nous aurions alors la construction la plus courante : $N / G N S D Y$ ). En revanche, dans l'exemple suivant, il y a bien substantivation de soy disant sergent, coordonné au nom prestre, le déterminant lesdiz étant mis en facteur commun ${ }^{(17)}$ :

(34) $[\ldots]$ il, estant en l'eglise de Saint-Mathurin de Larchant, environ heure de tierce, en la compaignie d'un gros prestre, court homme [...] et aussi d'un compaignon nommé Perrin, qui est un homme de moïen estat, à un visaige rondelet et un peu rousset, et lequel se disoit estre sergent du Chastellet de Paris, vit [...]; mais cedit jour, en disnant en ladite ville de Saint-Mathurin, il ouy dire et confesser ausdiz prestre et soy disant sergent qu'ilz avoient vuidié plusieurs bourses [...] (Registre criminel du Châtelet t.2, 1389-1392)

Selon les analyses ci-dessus, en moyen français SD ne présente aucune des caractéristiques des suites figées.

\subsection{Part du figement dans le passage de la séquence libre soi disant à l'unité du français contemporain}

\subsubsection{Figement}

Plusieurs changements relèvent du figement, entendu au sens large de « fixation $»$.

Sur le plan de la morphosyntaxe :

- le maintien de la forme soi, alors que l'évolution générale conduit à remplacer soi par se devant une forme verbale en -ant;

- la perte des possibilités d'insertion et de la coordination de disant: les deux éléments se sont soudés, ce qui correspond au figement dans les études sur la grammaticalisation.

Sur le plan sémantique, ce qui était une inférence suggérée, une interprétation provoquée par le cotexte ou le contexte, comme dans les exemples (27) à (29), à savoir la réserve du locuteur sur l'assertion rapportée, s'est intégré au sens de $\mathrm{SD}$; la sémantisation de cette inférence ${ }^{(18)}$ peut être envisagée comme un figement, en ce sens que désormais, en présence de SD, l'attitude du locuteur n'est plus interprétée à partir d'inférences tirées du cotexte ou de connaissances extralinguistiques, mais à partir de la seule occurrence de SD. Il y a donc enrichissement sémantique.

(17) Nous adoptons donc une autre analyse que dans Féron (à par.), où SD dans ce cas a été considéré comme adjectif; si nous excluons ici cette analyse pour le moyen français, c'est parce l'emploi adjectival de SD suppose un reparenthésage qui conduit à faire de SD une unité ( $c f$. infra, 3.2.2.), alors que SD Y apparait comme un groupe libre dans cet état de langue. Par ailleurs, cet emploi est rare en moyen français, et n'est pas attesté ensuite avant le français classique.

(18) Qui semble correspondre à ce que Perrin (2006 : 82) nomme « intégration de routines interprétatives associées à diverses formulations descriptivement affaiblies. » 


\subsubsection{Autres changements}

D'autres changements ne paraissent pas assimilables à un figement.

a) Réinterprétation sémantique

Dans l'usage non réflexif de SD tel que : « un hôtel, soi-disant luxueux » (où SD peut être paraphrasé par « qui est dit (à tort)»), le premier segment a perdu tout lien avec le pronom soi — ce que manifeste la graphie (stigmatisée) soit-disant ; SD a dans ce cas un sens passif ${ }^{(19)}$. Par conséquent, si, en moyen français, soi est coréférent du contrôleur de disant et donc permet l'assimilation de l'énonciateur du dire soit au référent du GN que le groupe $S D Y$ modifie (par ex, en 30), soit à l'être que dénote le $\mathrm{N}$, si $S D Y$ est déterminatif (par exemple en 31), soit au référent d'un GN présent dans le cotexte gauche (lorsque le groupe est en fin de proposition, comme dans (32) : «Thore, Dathan, et Abiron »), en français contemporain, la forme soi ne permet plus d'identifier un énonciateur; il en est de même lorsque SD est incident à un complément circonstanciel ou à une phrase ; seul le cotexte permet, parfois ( $c f$. 15 à 18), d'identifier l'énonciateur.

b) Réanalyse et recatégorisation

Dans la suite $N / G N S D Y$, SD Y a d'abord été un groupe (verbal) ayant pour mot tête disant; en français contemporain, c'est Y qui est le mot tête. Il s'agit ici d'une réanalyse, supposant un reparenthésage, qui aboutit à un figement : soi et Y sont, en moyen français, dans la dépendance de disant (le pronom pouvant être, on l'a vu, postposé), alors que par la suite les deux éléments soi et disant ont été sentis comme formant une unité et se sont soudés. Dès lors, il y a eu recatégorisation : de disant forme verbale régissant le pronom soi et $\mathrm{Y}$, on est passé à un adverbe (modalisateur, en raison du figement sémantique, c'est-à-dire de la sémantisation de l'inférence selon laquelle le locuteur doute du propos rapporté) intégrant la forme soi.

La recatégorisation en adjectif suppose de même un reparenthésage : la suite d'origine « pronom + disant » a été analysée comme une unité, incidente au nom qui suit. On notera que, si la dérivation participe présent $>$ adjectif est largement attestée, le fait remarquable ici est que l'adjectif inclut non la seule forme en -ant, mais aussi le pronom soi.

c) Extension des cotextes

La réinterprétation sémantique, la réanalyse et la recatégorisation ont pour conséquence une extension des cotextes :

— interprété comme passif (« qui est dit »), SD peut figurer derrière (« hôtel, soi-disant luxueux ») ou devant («soi-disant palace ») un nom dénué du trait /humain/ ;

- devenu adverbe modalisateur, SD peut apparaître dans des cotextes qui étaient impossibles pour la séquence pronom + disant, par exemple derrière un adjectif :

(35) $[\ldots]$ un beau couteau à légumes, inoxydable soi-disant (Beckett, Molloy, 1951)

(19) Cette réinterprétation sémantique peut être rapprochée du passage de voyant «qui voit » à «qui se voit» (une couleur voyante). 
devant un complément circonstanciel :

(36) Il a travaillé tout l'été, soi-disant pour s'acheter une nouvelle batterie. (Manoeuvre, L'Enfant du rock, 1985)

en tête de phrase (cf. supra, 4), ou de proposition (cf. supra, 18), à la finale absolue de la phrase :

(37) Tous cherchaient à nous protéger, soi-disant. (Schreiber, Un silence d'environ une demi-heure, 1996)

derrière le verbe ou entre le sujet et le verbe (cf. supra, 19), voire isolé entre deux points :

(38) Les filles qui avaient des grands volants comme des ailes, il les obligeait à s'envoler. Soi-disant. (Beck, La Prunelle des yeux, 1986)

S'y ajoute la possibilité pour SD d'être suivi d'une proposition en que, comme dans (17) ci-dessus.

\section{En guise de conclusion : bilan de la chronologie des changements}

\subsection{Données de corpus et descriptions lexicographiques}

L'analyse du corpus et le recours à des dictionnaires anciens ${ }^{(20)}$ permettent de repérer le moment où certains des changements décrits ci-dessus sont attestés — sans qu'ils soient nécessairement généralisés, des emplois anciens pouvant se maintenir à côté des emplois nouveaux : on rappellera ici la prescription normative qui restreint toujours aujourd'hui SD à l'usage réflexif, où soireste donc analysable comme pronom ${ }^{(21)}$.

Concernant le figement syntaxique, après le moyen français, l'ordre est fixe (soi est antéposé) et les deux éléments sont toujours contigus. L'indice le plus sûr du figement est néanmoins l'usage du trait d'union, qui signale que les deux éléments sont sentis comme formant une unité ; il parait imprudent de se fier aux éditions utilisées dans Frantext (certaines ajoutent un trait d'union là où l'édition originale n'en comporte pas), mais on note que le trait d'union apparait dans la première édition du Dictionnaire de l'Académie française (1694), quoique l'usage soit hésitant ${ }^{(22)}$ (on a un trait d'union dans un exemple figurant dans l'ar-

(20) A notre connaissance, le premier dictionnaire qui mentionne SD est celui de Furetière (1690).

(21) S'y ajoute un phénomène de persistance, définie comme la conservation « des traces de [la] classe de mots ou catégorie d'origine » (Marchello-Nizia 2006:41), à savoir le non-accord (selon la norme) de SD adjectif, justifié par le fait que, dans la construction originelle, $d i$ sant est un particpe présent.

(22) Si l'usage du trait d'union dès la première édition du Dictionnaire de l'Académie est le signe que SD est senti comme une unité, ce n'est que dans la sixième édition (1835) que SD donnera lieu à une entrée — quoiqu'il soit aussi décrit sous dire; auparavant, il figure sous l'entrée dire et/ou sous l'entrée soi. Ajoutons que dans le $T L F$, SD est traité à la fois dans un article à part et sous dire. 
ticle gentilhomme, ainsi que pour la sous-entrée soy-disant, sv. soy, mais non dans les exemples qui suivent).

La réinterprétation sémantique ( «qui se dit» > « qui est dit») est perceptible dans des emplois du type $N / G N S D$ Y ou Dét. $S D N$, et dans lesquels le N dénote un être du monde non doué de parole ; la première attestation date de 1729 (il s'agit aussi de la première attestation de la graphie soit-disant dans le corpus de Frantext, qui laisse penser que le premier élément n'est pas identifié comme un pronom) :

(39) [...] ils [la rose et le papillon] se jurent tous deux d'être unis à jamais. Le papillon content la quitte pour affaire : ne revient que sur le midi. Quoi! Ce feu soit-disant si vif et si sincére, lui dit la rose, est déja réfroidi ? Un siécle s'est passé, (c'étoit trois ou quatre heures) sans aucun soin que vous m'ayez rendu. (La Motte, Fables, 1719)

Cependant, l'usage non réflexif de SD avec un $\mathrm{N}$ dénotant un être doué de parole est antérieur ; Goosse (1991 : 429) note à propos de l'exemple suivant :

(40) [...] quand on revient de chez ces sorcières ou soi-disantes. (Sévigné, Correspondance, $t .2,1680)^{(23)}$

" A l'époque où il en coûtait chaud de se dire sorcière [...] il est douteux que l'expression doive être prise littéralement» (donc au sens de " celles qui se disent sorcières $»)$.

Cette occurrence nous fournit également le premier emploi de SD fonctionnant comme adjectif : la comparaison avec des exemples où le marqueur de rectification est suivi de SD et d'une seconde occurrence du N (« [...] avec la femme, ou soi-disant femme de mon confrère Vitulos ", Dulaurens, Le compère Mathieu, 1766, 143) conduit à analyser la suite Dét. Nou SD comme résultant d'une ellipse d'une seconde occurrence du N (« ces sorcières ou soi-disantes sorcières »). L'emploi comme adjectif antéposé n'est attesté dans le corpus qu'en 1743.

Pour ce qui est de la recatégorisation en adverbe, les premières occurrences significatives datent du dernier quart du XVIII ${ }^{\mathrm{e}}$ siècle. SD y occupe une position que ne pourrait pas occuper la forme en -ant de dire ; il peut par exemple être suivi d'un complément en pour :

(41) Grâce à la guerre qu'on fit déclarer, soi-disant pour achever la révolution, il nous en coûte déjà le sang d'un million d'hommes [...] (Desmoulins, Le Vieux cordelier $n^{\circ} 1 \grave{a} 6,1793$ )

On ajoutera que, dans la suite $N / G N S D Y$, l'accord n'est plus attesté après $1750^{(24)}$.

(23) C'est apparemment sur cet emploi que s'appuie Goosse (1991:429) pour affirmer que la «locution» soi-disant «naît au XVII ${ }^{\mathrm{e}}$ siècle quand il ne reste plus qu' un soi-disantcristallisé 》.

(24) Dans la première moitié du XVIII ${ }^{\mathrm{e}}$ siècle, disant dans la séquence $N / G N S D$ Y peut prendre la marque du masculin pluriel, comme c'est encore attesté à cette époque pour les participes présents (il n'y a en revanche pas d'accord lorsque le N est féminin, singulier ou pluriel). 
Il est plus difficile de repérer vers quel moment ce qui n'était qu'une inférence (la réserve du locuteur) a été codé en langue, attaché à la signification de SD ; certes, nous n'avons pas, après le moyen français, d'exemples où le cotexte indiquerait que le locuteur prend en charge l'assertion rapportée, alors qu'il est fréquent que le cotexte conduise à interpréter SD comme une marque de réserve de la part du locuteur. Mais en l'absence d'indice cotextuel, il parait impossible de savoir quelle signification avait SD pour les locuteurs. Nous disposons cependant d'un indice, à savoir les premières descriptions lexicographiques de SD : « On dit encore au Palais, soy disant, quand on parle d'une partie dont on conteste les qualitez » (Furetière 1690), « Terme de Palais, qu' on employe quand on ne veut pas convenir de la qualité que prend la personne contre qui l'on plaide. Un tel soy disant heritier, soy disant legataire, \&c. Il se dit aussi, Par raillerie ou par mespris. Un tel soy disant Docteur, soy disant Gentil-homme » (Dictionnaire de l'Académie française, 1694). A la fin du XVII siècle au moins, SD est donc pourvu d'une signification que n'a pas à priori se dire (que ces ouvrages ne mentionnent pas) : il fonctionne comme modalisateur.

\section{2. «Chronologie logique»}

Les données de corpus et les informations fournies par les dictionnaires ne permettent donc pas de repérer avec certitude des changements avant la fin du XVII ${ }^{\mathrm{e}}$ siècle ; pourtant, SD peut être considéré comme morphologiquement figé dès la deuxième moitié du XVI ${ }^{\mathrm{e}}$ siècle — à partir du moment où se devient de règle devant les formes en -ant. On peut supposer que si la forme soi s'est maintenue, c'est parce qu'à la forme soi disant était attaché un sens que n'avait pas se dire, à savoir l'idée de réserve du locuteur ${ }^{(25)}$.

SD devenu modalisateur a pu continuer dans un premier temps à s'employer comme forme verbale, distincte de se disant marqueur de discours rapporté. Progressivement, SD a cependant été senti comme une unité ( $c f$. supra, reparenthésage : disant [soi Y] > [soi disant]), dans laquelle le premier élément (soi) a pu perdre toute relation avec le pronom réfléchi ; SD a pu alors s'employer comme adverbe ou adjectif.

Les changements trouveraient donc leur origine dans la subjectivation, au sens que Traugott (1995:31-35), citée par Marchello-Nizia (2006:27), donne à ce terme : utilisation d'une forme non subjective à l'origine (qui ne dit rien de l'attitude du locuteur) pour véhiculer un commentaire du locuteur. Dans le cas de $\mathrm{SD}$, cette utilisation subjective résulte de la sémantisation d'une inférence possible dans certains cotextes, à savoir que le locuteur ne prend pas en charge l'assertion rapportée. C'est ce figement sémantique qui entrainerait le figement morphosyntaxique (maintien de soi et soudure des éléments) et permettrait la recatégorisation de SD en adverbe ou en adjectif.

(25) Une autre hypothèse pourrait être avancée pour expliquer le maintien de la forme $s o i$ audelà du XVI ${ }^{\mathrm{e}}$ siècle : SD, on l'a vu, apparait d'abord dans des textes juridiques, et il est signalé comme terme de palais/de pratique/de procédure dans le dictionnaire de Furetière, ceux de l'Académie française jusqu'à la huitième édition (1935) et celui de Littré. La langue juridique aurait conservé la forme ancienne du pronom, et cette forme serait passée dans la langue commune. 
Ce type d'évolution, qui conduit à la formation d'un modalisateur, est assimilable à une grammaticalisation au sens de Perrin $(2011: 86)^{(26)}$ : processus qui " consiste insensiblement à neutraliser le sens ascendant descriptif des expressions au profit d'un sens émergent énonciatif ou grammatical dépourvu de force descriptive au bout du compte ».

Au terme de l'évolution, alors qu'il y a perte de liberté pour les éléments qui constituent l'expression, la recatégorisation en adverbe modalisateur entraine davantage de liberté syntaxique, cette autonomisation par rapport aux constituants de la phrase étant observée de façon générale dans l'évolution qui conduit d'un adverbe de constituant à un adverbe modalisateur ( $c f$. par exemple Combettes \& Kuyumcuyan : 2007, à propos de en vérité, à la vérité).

(26) Perrin (2011) utilise aussi pour décrire ce phénomène le terme de «figement formulaire». 


\section{Bases textuelles}

7FMR (Corpus de Textes de Français Médiéval et de la Renaissance). http://atilf.atilf.fr/scripts/mep.exe ?CRITERE=TEXTES_2012；ISIS =isis_dmf.txt

Frantext. http://www.frantext.fr

\section{Dictionnaires}

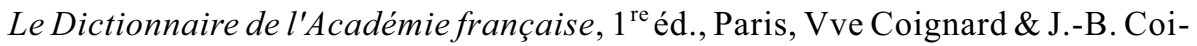
gnard, 1694.

Dictionnaire de l'Académie française, $6^{\mathrm{e}}$ éd., Paris, Firmin-Didot frères, 1835.

Dictionnaire de l'Académie française, $8^{\mathrm{e}}$ éd. Paris, Librairie Hachette, 1932-35.

Dictionnaire du Moyen Français, version 2012. ATILF CNRS - Université de Lorraine. www.atilf.fr/dmf $[D M F]$

Dubois, J. dir. (1998) : Dictionnaire de la langue française, Lexis, Paris, Larousse. [Lexis]

FURETIÈRE, A. (1690) : Dictionnaire universel, A. \& R. Leers, La Haye / Rotterdam.

IMBS, P. puis QUEMADA, B. dir. (1971-1994) : Trésor de la langue française, dictionnaire de la langue du XIX et du XX $X^{e}$ siècle (1789-1960), Paris, éd. du CNRS [puis] Gallimard. http ://atilf.atilf.fr/tlf.htm

REY-DeBove, J. \& REY, A. dir. (2012) : Le nouveau petit Robert, Paris, Le Robert. $[N P R]$

LitTRÉ, E. (1863-1872) : Dictionnaire de la langue française, Paris, Hachette.

\section{Bibliographie}

AuthIER-REVUZ, J. (1995) : Ces mots qui ne vont pas de soi. Boucles réflexives et non-coïncidences $d u$ dire, tome 1, Paris, Larousse.

Combettes, B. \& KuyumcuyAn, A. (2007) : « La formation des modalisateurs en français : le cas des locutions formées sur vérité », Langue française 156, 76-92.

Combettes, B. \& MARChello-Nizia, C. (2010) : « La périodisation historique. Le cas du français préclassique ». In : Combettes, B. e.a., Le changement en français. Etudes de linguistique diachronique, Bern/Berlin/Bruxells/Frankfurt/New York/Oxford/Vienne, Peter Lang, 129-141.

FÉRON, C. (à paraitre) : Soi-disant : étude diachronique, Actes du colloque Diachro 6, Leuwen, 17-19 octobre 2012.

FOURNIER, N. (1998) : Grammaire du français classique, Paris, Belin.

Goosse, A. (1991) : Mélanges de grammaire et de lexicologie françaises, Leuven / Louvain-la-neuve, Peeters et Publications linguistiques de Louvain. - (2008) : Le bon usage, $14^{\mathrm{e}}$ édition, Bruxelles, Duculot.

GuimiER, C. (1988) : Syntaxe de l'adverbe anglais, Villeneuve d'Ascq, Presses universitaires de Lille. 
- (1996) : Les adverbes du français. Le cas des adverbes en -ment, Gap/Paris, Ophrys.

GROSS, G. (1996) : Les expressions figées en français, noms composés et autres locutions, Gap/Paris, Ophrys.

LeCOLle, M. (2006) : « Changement dans le lexique — changement du lexique : Lexicalisation, figement, catachrèse », Cahiers de praxématique 46, 23-46.

MARCHELlO-NiZIA, C. (1979) : Histoire de la langue française aux XIVe et XVe siècles, Paris, Bordas.

— (2006) : Grammaticalisation et changement linguistique, Bruxelles, De Boeck.

MARTIN, R. (1987) : Langage et croyance, Bruxelles, Mardaga.

- (1992) : Pour une logique du sens, $2^{\mathrm{e}}$ éd., Paris, PUF.

MARTin, R. \& Wilmet, M. (1980) : Syntaxe du moyen français, Bordeaux, SOBODI.

NoAilly, M. (1999) : L'adjectif en français, Gap/Paris, Ophrys.

NøJGAARD, M. (1992-1993-1995) : Les adverbes français. Essai de description fonctionnelle, Copenhague : Munksgaard.

PERRIN, L. (2006) : «Énonciation, grammaticalisation et lexicalisation », Cahiers de praxématique 46, 81-101.

- (2011) : «Figement, énonciation et lexicalisation citative ». In :Anscombre, J.-C. \& Mejri, S. dir., Le figement linguistique : la parole entravée, Paris, Champion, 81-94.

RIEGEL, M. e.a. (2009) : La grammaire méthodique du français, Paris, PUF.

ROSIER, L. (1999) : Le discours rapporté. Histoire, théories, pratiques, Paris Bruxelles, De Boeck \& Larcier, Département Duculot.

SCHNEDECKER, C. (2002) : «Présentation : les adjectifs “inclassables”, des adjectifs du troisième type ? », Langue française 136, 3-19.

TRAUGOTT, E. C. (1995) : «On the rise of epistemic meaning in English : an example of subjectification in semantic change », Language 65, 31-55. 